\title{
Bruton's Disease Presenting With Arthritis; A Case Report
}

\author{
Atena Ramezanali Yakhchali ${ }^{1}$; Zahra Chavoshzadeh ${ }^{2}$; Mehrnaz Mesdaghi ${ }^{3}$; Mahboubeh \\ Mansouri ${ }^{3}$; Reza Shiari ${ }^{4, *}$ \\ ${ }^{1}$ School of Medicine, Shahid Beheshti University of Medical Sciences, Tehran, IR Iran \\ 2 Pediatric Infections Research Center, Mofid Children's Hospital, Shahid Beheshti University of Medical Sciences, Tehran, IR Iran \\ 3 Department of Immunology, Mofid Children's Hospital, Shahid Beheshti University of Medical Sciences, Tehran, IR Iran \\ Department of Immunology, Mofid Children's Hospital, Shahid Beheshti University of Medical Sciences, Tehran, IR Iran
${ }^{4}$ Department of Pediatric Rheumatology, Mofid Children's Hospital, Shahid Beheshti University of Medical Sciences, Tehran, IR Iran \\ ${ }^{*}$ Corresponding author: Reza Shiari, Department of Pediatric Rheumatology, Mofid Children's Hospital, Shahid Beheshti University of Medical Sciences, Tehran, IR Iran. Tel/Fax: +98- \\ 2122227033, E-mail: shiareza@yahoo.com
}

Received: June 10, 2014; Accepted: July 24, 2014

\begin{abstract}
Introduction:X-linked Agammaglobulinemia (XLA) is one of the primary humoral immunodeficiencies. It usually presents symptoms of recurrent infections, but in some unusual cases it may present rheumatologic manifestations.

Case Presentation:The current paper presents the cases of two boys with arthritis treated for juvenile rheumatoid arthritis (JRA) without proper responses. Addition of some recurrent infections in the course of their disease led to work-up them for immunodeficiencies.

Conclusions: According to the results of these work-ups, XLA was diagnosed for the cases.

Keywords: Agammaglobulinemia; Arthritis; Agammaglobulinemia
\end{abstract}

\section{Introduction}

X-Linked agammaglobulinemia(XLA or Bruton's disease) is a subset of primary immunodeficiencies characterized by a defect in B-cell maturation (1). Most of the patients with hypo or agammaglobulinemia present symptoms of the recurrent bacterial respiratory infections, but XLA may also present symptoms of arthritis, which can be the only manifestation of the disease or its complication (2); therefore, recurrent arthritis needs a high index of suspicion to be diagnosed as XLA. The current paper reports two cases of arthritis as a manifestation of this condition, and discusses their clinical courses.

\section{Case Presentation}

The first case was a six-year-old boy from non-consanguineous parents with no siblings. The first presentation, at the age of three years, was arthritis in both knees diagnosed as Juvenile Rheumatoid Arthritis (JRA) according to the serial work-ups and treated with prednisolone, colchicine, and Ebetrex. However, the patient had several flare-ups despite treatment. During the course of the disease, he experienced several episodes of diarrhea. About a year later, signs and symptoms of acute sinusitis showed up, and were confirmed by spiral computerized tomography scan (CT-scan) of paranasal sinuses, illustrating mastoiditis and pansinusitis. At that time, broad-spectrum antibiotics were administered, resulting almost in no improvement in the patient's condition. Resistant respiratory infections and histories of recurrent arthritis raised a suspicion of an underlying disease. After an immunologic work-up and further evaluation (Table 1), diagnosis of Bruton's disease was made and intravenous immunoglobulin (IVIG) with dosage of $600 \mathrm{mg} / \mathrm{kg}$ per month was started. Shortly after the new treatment and also discontinuation of the previous drugs, his symptoms began to resolve. The other case was a 12-year-old boy from non-relative parents with no siblings. Eight years ago, at the age of four, due to pauciarticular arthritis and following work-ups, diagnosis of JRA was made and the patient was discharged with prednisolone, hydroxychloroquine, and sulfasalazine. Three years later, the patient presented symptoms of exacerbation of the disease, poorly responsive to immunosuppressive agents. On the admission, lab results demonstrated a flare-up of the arthritis. After several admissions, addition of recurrent upper respiratory tract infections, otitis media and developing non-specific coughs made the condition more ominous. Chest spiral CT-scan showed opacities in lingula and peribronchial regions of the both lungs. History of flaccid paralysis followed by the polio vaccine administration at the age of six months, history of an immunodeficiency disorder in the patient's maternal uncle, and poor response to broad-spectrum antibiotics were clues to work up the patient for primary immunodeficiencies (Table 2). According to the results, Bruton's disease was diagnosed. IVIG with dosage of 600 $\mathrm{mg} / \mathrm{kg}$ were prescribed and symptoms relieved. 
Table 1. Immunologic Work-up and Flow Cytometry Results (Patient 1)

\begin{tabular}{lc}
\hline & Results \\
\hline IgM (nephelometry), mg/dL & not detected \\
IgG (nephelometry), mg/dL & not detected \\
\hline IgA (nephelometry), mg/dL & 9 \\
\hline IgE (ELISA), IU/mL & 3 \\
\hline Anti-tetanus IgG (ELISA), IU/mL & undetectable \\
\hline Anti-diphtheria IgG(ELISA), IU/mL & undetectable \\
\hline Isohemagglutinin anti A, Titer & $<1 / 2$ \\
\hline Isohemagglutinin anti B, Titer & $<1 / 2$ \\
\hline Flowytometry & \\
\hline CD3, \% & 78.1 \\
\hline CD4, \% & 38.6 \\
\hline CD8, \% & 33.6 \\
\hline CD16, \% & 11.3 \\
\hline CD19, \% & $0.2(l o w)$ \\
\hline CD56, \% & 9.2 \\
\hline CD4/CD8 & 1.15 \\
\hline
\end{tabular}

Table 2. Immunologic Work up and Flow Cytometry Results (Patient 2)

\begin{tabular}{lc}
\hline & Results \\
\hline IgM (nephelometry), mg/dL & not detected \\
IgG (nephelometry), mg/dL & not detected \\
\hline IgA (nephelometry), mg/dL & 3 \\
\hline IgE (ELISA), IU/mL & $<0.1$ \\
\hline Anti-tetanus IgG(ELISA), IU/mL & undetectable \\
\hline Anti-diphtheria IgG(ELISA), IU/mL & undetectable \\
\hline Isohemagglutinin anti A, Titer & $<1 / 2$ \\
\hline Isohemagglutinin anti B, Titer & $<1 / 2$ \\
\hline Flowytometry & \\
\hline CD3, \% & 90.5 \\
\hline CD4, \% & 42.4 \\
\hline CD8, \% & 47.6 \\
\hline CD16, \% & 6.7 \\
\hline CD19, \% & $2(l o w)$ \\
\hline CD56, \% & 5.4 \\
\hline CD4/CD8 & 0.89 \\
\hline
\end{tabular}

\section{Discussion}

XLA is a humoral immunodeficiency, characterized by a defect in B-cell maturation followed by marked reduction in all of the isotypes of serum immunoglobulins as a result of BTK-gene mutation on X-chromosome (1, 3, 4). Delay in diagnosis of primary immunodeficiencies (PIDs) is usual and these disorders are more common than thought (4). Since 1952, when Bruton's disease was first described, the prevalence of infections and frequency of hospital admissions have decreased, because of earlier diagnosis and proper management (3). Recurrent infections are the main manifestations of PIDs $(3,5)$, especially sinopulmonary ones (6), but other infections such as meningitis, arthritis, osteomyelitis, cellulitis, and gastroenteritis may occasionally occur (6). In common variable immunodeficiency (CVID) and XLA, compared to the other congenital immunodeficiencies, bone and joint infections are observed more $(6,7)$. Nonpathogenic organisms in immunocompetent individuals, like mycoplasma and ureaplasma, are the most common agents causing arthritis in such immunocompromised patients (6), although Staphylococcus aureus and streptococcal infections are also commonly observed $(3,6,7)$. Bone and joint abnormalities can occur in patients with PID, with arthritis being the most common joint manifestation. The type of arthritis is usually aseptic oligo/ mono arthritis, which can be even the sole presentation $(2,7,8)$. For many years, it was believed that hypogammaglobulinemia and agammaglobulinemia can be associated with symmetric polyarthritis with occasional extra-articular manifestations, resembling RA (7, 9). A report by Wang XC investigated this issue. In his study, which was conducted on eight patients with suspected XLA, results showed that three of eight had polyarthritis without any evidence of infection (10). In another study conducted on 281 patients with immunodeficiency, 30 had arthritis as the first presentation, which was more common in individuals with Bruton's disease (22\%). Nonspecific arthritis was more common than septic type. Monoarthritis and oligoarthritis were observed in Bruton's disease and CVID, respectively and more commonly arthritis can get septic as a complication of an underlying arthritis due to delayed diagnosis (9). In conclusion, patients with recurrent arthritis or resistant ones should be assessed for immunodeficiency disorders, especially when other infectious states accompanying during the course of the disease; therefore, awareness of physicians concerning this issue would certainly prevent serious and life-threatening complications in this group of patients.

\section{Authors' Contributions}

Reza Shiari: attending physician; Zahra Chavoshzadeh: attending physician; Mehrnaz Mesdaghi: laboratory diagnosis and editing the manuscript; Mahboubeh Mansouri: consultant physician; Atena Ramezanali Yakhchali: writing the manuscript.

\section{References}

1. Dan L, Anthony F, Dennis K, Stephen H, Jameson J, Joseph L. Harrison's Principles of Internal Medicine. 18th ed: McGraw Hill Professional; 2011.

2. Hansel TT, Haeney MR, Thompson RA. Primary Hypogammaglobulinemia and Arthritis. BrMed J.1987;295(6591):174-5. 


\section{Shiari R et al.}

3. Chun JK, Lee TJ, Song JW, Linton JA, Kim DS. Analysis of clinical presentations of Bruton disease: a review of 20 years of accumulated data from pediatric patients at Severance Hospital. Yonsei Med J. 2008;49(1):28-36.

4. Melo KM, Dantas E, De Moraes-Pinto MI, Condino-Neto A, Gonzalez IG, Mallozi MC, et al. Primary Immunodeficiency May Be Misdiagnosed as Cow's Milk Allergy: Seven Cases Referred to a Tertiary Pediatric Hospital. ISRN Pediatr. 2013;2013:470286.

5. Ariganello P, Angelino G, Scarselli A, Salfa I, Della Corte M, De Matteis A, et al. Relapsing Campylobacter jejuni Systemic Infections in a Child with X-Linked Agammaglobulinemia. Case Rep Pediatr. 2013;2013:735108.

6. Bloom KA, Chung D, Cunningham-Rundles C. Osteoarticular infectious complications in patients with primary immunodeficiencies. Curr Opin Rheumatol. 2008;20(4):480-5.

7. Sordet C, Cantagrel A, Schaeverbeke T, Sibilia J. Bone and joint disease associated with primary immune deficiencies. Joint Bone Spine. 2005;72(6):503-14.

8. Peters TR, Brumbaugh DE, Lawton AR, Crowe JJ. Recurrent pneumococcal arthritis as the presenting manifestation of X-linked agammaglobulinemia. Clin Infect Dis. 2000;31(5):1287-8.

9. Verbruggen G, De Backer S, Deforce D, Demetter P, Cuvelier C, Veys E, et al. X linked agammaglobulinaemia and rheumatoid arthritis. Ann Rheum Dis. 2005;64(7):1075-8.

10. Wang XC. [Clinical features of X-linked agammaglobulinemia: analysis of 8 cases]. Zhonghua Er Ke Za Zhi. 2004;42(8):564-7. 